\title{
JEAN DE SMET
}

\section{Le problème des signes de Thurstone. Une application de la méthode SEP}

Revue française d'automatique, d'informatique et de recherche opérationnelle. Recherche opérationnelle, tome 2, no V2 (1968), p. 33-46.

$<$ http://www.numdam.org/item?id=RO_1968_2_2_33_0>

(C) AFCET, 1968, tous droits réservés.

L'accès aux archives de la revue « Revue française d'automatique, d'informatique et de recherche opérationnelle. Recherche opérationnelle » implique l'accord avec les conditions générales d'utilisation (http://www.numdam.org/ legal.php). Toute utilisation commerciale ou impression systématique est constitutive d'une infraction pénale. Toute copie ou impression de ce fichier doit contenir la présente mention de copyright.

\section{Numdam}

Article numérisé dans le cadre du programme

Numérisation de documents anciens mathématiques

http://www.numdam.org/ 
R.I.R.O.

(2e année, No 11, 1968, p. 33-46)

\title{
LE PROBLEME DES SIGNES DE THURSTONE Une application de la méthode SEP
}

\author{
par Jean De Smet $\left({ }^{1}\right)$
}

Résumé. - L'objet de cet article est de présenter un exemple d'application de la méthode de séparation et évaluation progressives.

Le problème consiste en un programme quadratique en nombres entiers provenant de la méthode centroïde d'analyse factorielle de Thurstone.

ll s'énonce : Maximiser une forme quadratique dont les pariables sont binaires, de module un.

La non-convexité de la fonction économique dans le domaine formé par l'enveloppe convexe des solutions réalisables nécessite une méthode d'optimisation globale. La procédure SEP permet de résoudre le problème grâce à l'introduction d'une fonction majorante dérivant des normes d'une matrice.

Le problème peut être transposé sur un graphe et trouve ainsi d'autres applications.

\section{INTRODUCTION. ORIGINE DU PROBLEME}

La méthode de séparation et évaluation progressive (réf. [4], [5]) a permis de résoudre un grand nombre de problèmes d'optimisation à caractère combinatoire. Le présent article a pour but d'en donner un exemple dans le cas d'un programme quadratique simple. Le problème traité a trouvé son origine en analyse factorielle (réf. [1], [2]).

L'analyse factorielle est une branche de la statistique qui traite de la structure des matrices de covariance. Son modèle principal est un modèle linéaire par lequel on tente d'expliquer les corrélations entre les variables étudiées par un nombre aussi petit que possible de facteurs linéaires. Le but de la recherche est de déterminer quel est le nombre minimum de facteurs indépendants nécessaires et quels sont les influences des facteurs sur chacune des variables ou encore quels sont les coefficients

(1) Centre de Recherche pour la Défensẹ, Bruxelles. 
factoriels. Dans ce modèle principal on ne s'intéresse qu'aux covariances et non aux variances : l'ensemble des facteurs ne détermine pas nécessairement chaque variable; il suffit que les variables résiduelles obtenues après extraction des facteurs soient non corrélées.

La méthode centroïde est la méthode la plus ancienne d'analyse factorielle. Elle est due à Thurstone (1930-31). Dans son principe elle fait appel à une correspondance formelle entre la covariance d'un couple de variables et le produit scalaire de 2 vecteurs dans un espace vectoriel de dimension égale au nombre de facteurs, qui y forment une base orthonormale. Les facteurs sont recherchés selon un certain ordre d'importance.

Le premier facteur correspond au vecteur unité orienté selon la direction centrale du faisceau des vecteurs correspondant aux variables. Le second facteur correspond au vecteur unité orienté selon la direction centrale du faisceau projeté dans l'hyperplan perpendiculaire au premier facteur et ainsi de suite. La direction centrale du faisceau est celle d'un vecteur résultant d'une somme algébrique des vecteurs correspondant aux variables. La direction centrale optimale est celle du vecteur-somme le plus grand en module. Cet optimum est obtenu grâce au choix judicieux des signes qui interviennent dans la somme algébrique des vecteursvariables. On est ainsi amené à résoudre un problème d'optimisation quadratique en nombres entiers $(+1$ et -1$)$. Mais la fonction économique du programme n'est ni concave ni convexe dans le domaine élargi de l'enveloppe convexe des solutions réalisables.

\section{POSITION DU PROBLEME RESTREINT ET SOLUTION SOUS-OPTIMALE CLASSIQUE}

Soit une matrice de covariance $B(n \times n$, symétrique). Quel esí le vecteur "signe " $q$, composé d'éléments $q_{i}$ valant +1 ou $-1(i=1 \ldots$ à ...n $)$ qui maximise la fonction économique quadratique $q^{\prime} B q$ ? Les éléments de la diagonale principale de $B$ n'interviennent pas dans la détermination de l'optimum car pour toute solution admissible l'apport de ces éléments diagonaux à la fonction économique est trace $(B)$. Le problème reste donc inchangé, si on suppose que la diagonale principale de $B$ est nulle.

L'algorithme employé dans la méthode centroïde classique assure en fait un optimum local du problème posé. J'entends par optimum local une solution telle qu'aucune autre solution qui n'en diffère que par le signe d'un des éléments $q_{i}$, n'est meilleure que la solution proposée.

L'algorithme de Thurstone est le suivant :

Initialement on choisit un vecteur signe arbitraire.

Comme $q^{\prime} B q=\sum_{i=1}^{n} q_{i} B_{i} q$, où $B_{i}$ est la $i^{\mathrm{e}}$ ligne de la matrice $B$, on examine chaque somme $s_{i}=q_{i} B_{i} q$. Si toutes ces sommes $s_{i}$ sont non 
négatives, l'optimum local est atteint par le vecteur signe considéré. S'il en existe qui sont négatives, on change le signe du $q_{i}$ correspondant au $s_{i}$ le plus petit et on reprend l'examen.

L'algorithme prend nécessairement fin quand tout $s_{i} \geqslant 0$. Il est aisé de vérifier sur des matrices de taille minimum $4 \times 4$ que cette procédure ne conduit pas nécessairement à l'optimum global.

\section{LE PROBLEME DES SIGNES ELARGI}

Considérons le problème suivant :

Maximiser $x^{\prime} A x+b^{\prime} x$ sous la condition

$$
c \leqslant x \leqslant d
$$

Supposons que dans cette expression la matrice $A$ est à diagonale principale non négative $\left(a_{i i} \geqslant 0\right.$ pour tout $\left.i\right)$.

Un changement de variables linéaire transforme ce problème par :

$$
\begin{aligned}
& q_{i}=\left(x_{i}-\frac{c_{i}+d_{i}}{2}\right) \frac{2}{d_{i}-c_{i}} \quad(i=1 \ldots n) \\
& q_{0}=1
\end{aligned}
$$

en : maximiser $q^{\prime} B q$

$$
\text { sous } \begin{aligned}
-1 \leqslant q_{i} & \leqslant 1 \quad(i=1 \ldots n) \\
q_{0} & =1
\end{aligned}
$$

ou $B$ est une matrice $(n+1) \times(n+1)$ dont les éléments sont

$$
\begin{aligned}
& b_{i j}=\left(\frac{d_{i}-c_{i}}{2}\right)\left(\frac{d_{j}-c_{j}}{2}\right) a_{i j} \quad(i \text { et } \jmath \neq 0) \\
& b_{0 j}=\sum_{i=1}^{n}\left(\frac{d_{i}+c_{i}}{2}\right)\left(\frac{d_{j}-c_{j}}{2}\right) a_{i j}+b_{j}\left(\frac{d_{j}-c_{j}}{4}\right) \\
& b_{i 0}=\sum_{j=1}^{n}\left(\frac{d_{i}-c_{i}}{2}\right)\left(\frac{d_{j}-c_{j}}{2}\right) a_{i j}+b_{i}\left(\frac{d_{i}-c_{i}}{4}\right) \\
& b_{00}=0
\end{aligned}
$$

La matrice $B$ est symétrique et à diagonale principale non négative si tel est le cas de la matrice $A$. La symétrie n'est d'ailleurs pas requise car le problème ne change pas par symétrisation. 
On peut encore élargir le problème en permettant :

$$
-1 \leqslant q_{0} \leqslant 1
$$

Par application du théorème de Farkas généralisé (réf. [3]) on peut aisément démontrer que tout optimum local de ce problème est en un sommet de l'hypercube $-1 \leqslant q_{i} \leqslant 1$.

Dans la démonstration la condition $b_{i i} \geqslant 0$ pour tout $i$ est essentielle.

Les conditions d'optimum local du problème élargi, fournies par le théorème de Farkas, ne sont autres que les conditions d'optimum local de Thurstone. La condition supplémentaire $q_{0}=1$, n'est pas limitative car $q^{\prime} B q=(-q)^{\prime} B(-q)$ et des deux solutions équivalentes $q$ et $-q$ il suffit de choisir celle qui assure $q_{0}=1$. La solution du problème élargi est donc identique à celle du problème restreint. Il a simplement fallu introduire une variable supplémentaire. Le programme quadratique est réduit à un problème de combinatoire.

\section{LA METHODE S.E.P.}

La méthode de Séparation et Evaluation Progressive a été décrite dans plusieurs articles (réf. [4], [5]).

On n'en donne ici qu'un aperçu adapté au problème des signes.

Soit un problème d'optimisation : déterminer $\hat{x}$ qui maximise $f(x)$ sous un ensemble de conditions limitant les $x$ réalisables à un ensemble fini $\xi$.

Il est parfois nécessaire de considérer un ensemble $\bar{\xi}$ plus large que $\xi$. Cet onsemble $\bar{\xi}$ comprend toutes les solutions réalisables mais peut-être aussi d'autres solutions $(\xi \subset \bar{\xi})$

Dans la collection $\xi$ des ensembles de solution $C$, la recherche de $\hat{x}$ est la recherche d'un ensemble $\hat{C}$ membre de la collection qui ne comprend qu'une seule solution réalisable et tel que $f(\hat{C}) \geqslant f(x)$ pour $\forall x \in \xi$. L'ensemble $\hat{C}$ correspond à la solution $\hat{x}$.

Le principe de la méthode est le suivant:

- on sépare $\bar{\xi}$ en morceaux,

- on évalue pour chaque morceau $C \subseteq \bar{\xi}$ une valeur par excès $\varphi$ $\operatorname{des} f(x) / x \in C$,

- on recommence.

La séparation des ensembles en sous-ensembles peut être représentée par une arborescence $H(S, \Gamma)$.

La racine $s_{0}$ de cette arborescence correspond à l'ensemble $\bar{\xi}$.

Tout sommet $s$ correspond à un ensemble $C \subseteq \bar{\xi}$, qui possède un seul précédent si $s \neq s_{0}$. 
La séparation doit respecter les axiomes suivants :

i) Axiome de réunion : $C(s)=\bigcup_{t \in \Gamma(s)} C(t)$.

On ne perd pas de solutions.

ii) Axiome de finitude : $|S|<\infty$. Le nombre de sommets est fini.

iii) Axiome d'arrêt : Si $\Gamma(s)=\varnothing,|C(s)| \leqslant 1$. Un sommet terminal comprend soit une solution unique identifiable $|C(s)|=1$, soit pas de solution $|C(s)|=0$.

La fonction d'évaluation est une fonction d'ensemble $\varphi(C)$ appelée fonction majorante qui est une évaluation par excès de toute solution réalisable $x \in C$.

$$
\varphi(C) \geqslant f(x) \quad \text { pour } \quad \forall x \in C
$$

et qui coïncide avec la fonction $f(x)$ quand l'ensemble $C$ comprend uniquement la solution réalisable $x$ :

$$
\varphi(C)=f(x) \quad \text { si } \quad C=\{x\}, x \in \xi
$$

ou si $s(C)$ est tel que $\Gamma(s)=\varnothing$.

Un sommet $s$ peut donc être de l'un des trois types suivants :

i) $\Gamma(s) \# \varnothing$ soit $|\Gamma(s)|=r$ et pour les $r$ sommets $t \in \Gamma(s)$, on peut calculer $v(t)$. L'ensemble $C(s)$ peut être décomposé en $r$ sousensembles $C^{(1)} \ldots C^{(r)}$ pour lesquels $\varphi\left(C^{(k)}\right)$ peut être calculé.

ii) $\Gamma(s)=\varnothing$ et $|C(s)|=1$ : l'ensemble $C(s)$ ne comprend qu'une seule solution réalisable $x$ et alors $\varphi(C)=f(x)$.

iii) $\Gamma(s)=\varnothing$ et $|C(s)|=0$ : l'ensemble $C(s)$ ne comprend pas de solution réalisable.

L'algorithme est le suivant :

(1) On considère $s_{0}$ correspondant à $\bar{\xi}$. On calcule $\varphi\left(s_{0}\right)=\varphi(\bar{\xi})$.

On constitue la liste $Z$ de sommets comprenant le sommet $s_{0}$.

(2) On parcourt la liste $Z$ des sommets. Si la liste est vide, il n'existe pas de solution et l'on s'arrête, sinon l'on passe à (3).

(3) On choisit le sommet $s$ pour lequel $\varphi(s)$ est le plus grand.

(4) Pour ce sommet $s$ on examine le type :

- s'il est de type (i) on passe au point (5),

- s'il est de type (ii), il correspond à $\hat{C}$ et donc à $\hat{x}$. Le calcul s'arrête et $\varphi(s)=f(\hat{x}), s=\hat{s}$,

- s'il est de type (iii) le sommet $s$ est exclu de la liste $Z$ et l'on retourne à (2).

(5) On détermine selon un certain critère les suivants de $s$ pour lesquels on calcule la fonction majorante. 
(6) Le sommet $s$ est exclu de la liste des sommets. Tous les sommets $t \in \Gamma(s)$ (suivants de $s$ ) sont ajoutés à la liste $Z$. L'on retourne à $(2)$.

Cet algorithme assure bien que l'on trouve la solution $\hat{x}$ en un nombre de pas fini, car à toute itération $\varphi(s) \geqslant \varphi(t)$ pour $\forall t \in Z$.

et si $s=\hat{s}, f(\hat{x})=\varphi(\hat{s})>\varphi(t)>f(x)$ pour $\forall x \in \xi$ car aucune solution n'a été perdue.

On ne parcourt pas toute l'arborescence pour trouver la solution. La liste $Z$ ne comprend à la dernière itération que des sommets $t$ pour lesquels $\varphi(t) \leqslant f(\hat{x})$ et qui sont suivants immédiats de sommets $s$ pour lesquels $\varphi(s) \geqslant f(\hat{x})$.

\section{APPLICATION DE LA METHODE SEP AU PROBLEME DES SIGNES}

\section{a) Enoncé}

On cherche $\hat{q}$ qui maximise $q^{\prime} B q$ sous $q_{i}=+1$ ou -1 . $B$ est une matrice symétrique à diagonale nulle $(n \times n)$. L'ensemble $\xi=\left(q_{i}=+1\right.$ ou $-1 / i \in J)$ est l'ensemble des solutions réalisables.

L'ensemble $J=(1,2, \ldots, n)$ est l'ensemble des indices.

\section{b) Construction des ensembles $C$}

Soit $I \subset J$ un ensemble d'indices pour lequel un choix a été établi.

$q_{i}=p_{i} / i \in I$ où $p_{i}$ est une valeur fixe +1 ou -1 pour l'ensemble $C$ considéré

$\bar{I}=J / I$ est l'ensemble des autres indices.

$$
C=\left\{\left(q / q_{i}=p_{i} / i \in I\right) \cup\left(q_{i}=+1 \text { ou }-1 / i \in \bar{I}\right)\right\}
$$

- Il n'existe pas d'ensembles $C$ ne comprenant pas de solutions et $\xi=\bar{\xi}$.

$\rightarrow$ Si $I=\varnothing, C=\xi=\bar{\xi}$

- Si $I=J, \bar{I}=\varnothing, C=\left\{q / q_{i}=p_{i} / i \in J\right\}$ l'ensemble $C$ comprend une solution unique $q=p$.

- Si $I \subset J$ strictement, tout ensemble $C$ a deux sous-ensembles $C^{(1)}$ et $C^{(2)}$ qui sont suivants immédiats de $|\bar{I}|$ façons différentes.

$$
\begin{gathered}
C^{(1)}=\left\{\left(q / q_{i}=p_{i} / i \in I\right) \cup\left(q_{j}=+1 / j \in \bar{I}, j \text { unique }\right)\right. \\
\cup\left(q_{k}=+1 \text { ou }-1 / k \in \bar{I} /(j)\right\} \\
\begin{aligned}
C^{(2)}=\left\{q /\left(q_{i}=p_{i} / i \in I\right) \cup\left(q_{j}=-1 / j \in \bar{I}, j\left(C^{(2)}\right)=j\left(C^{(1)}\right)\right.\right. \\
\cup\left(q_{k}=+1 \text { ou }-1 / k \in \bar{I} /(j)\right\}
\end{aligned}
\end{gathered}
$$

et $I^{\prime}=I \cup(j)$ est le nouvel ensemble d'indices pour $C^{(1)}$ et $C^{(2)}$. 
L'arborescence $H(S, \Gamma)$ comprend alors $n+1$ lignes de sommets numérotés de 0 à $n$. Il y a $2^{k}$ sommets à la ligne $k$. Cette ligne $k$ comprend tous les suivants en $k$ pas de $s_{0}$. Il y a donc en tout $2^{n+1}-1$ sommets dont $2^{n}$ à la $n^{\mathrm{e}}$ ligne, correspondant à des solutions uniques.

\section{c) La fonction majorante}

La fonction majorante est la suivante :

$$
\varphi(C)=2\left(\sum_{i \in I} \sum_{\substack{j \in I \\ j>i}} b_{i j} p_{i} p_{j}+\sum_{i \in \bar{I}}\left|\sum_{j \in I} b_{i j} p_{j}\right|+\sum_{i \in \bar{I}} \sum_{\substack{j \in \bar{I} \\ j>i}}\left|b_{i j}\right|\right)
$$

Elle peut être visualisée sur la matrice $B$

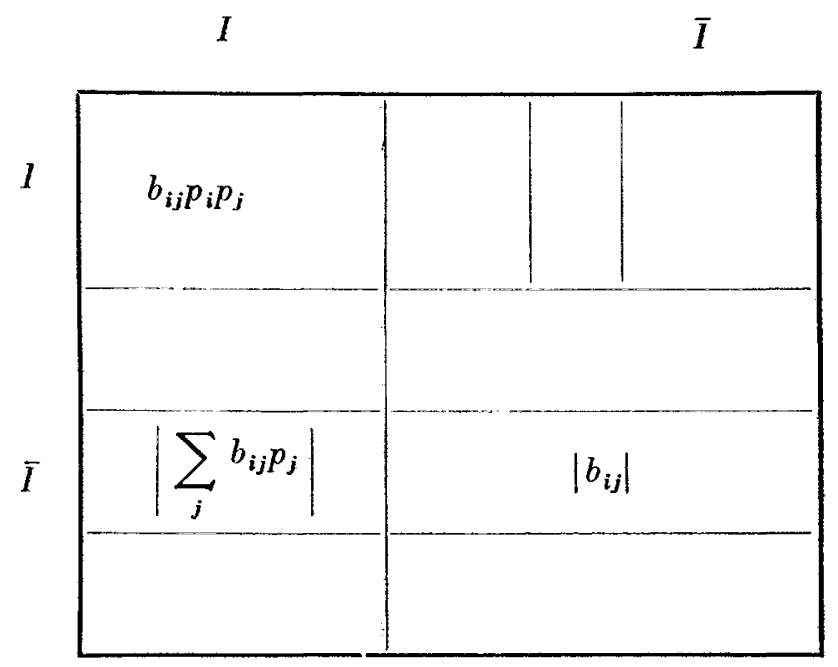

On vérifie aisément que cette fonction remplit les conditions énoncées au paragraphe 4 .

On a que $\varphi(\xi)=2 \sum_{i \in J} \sum_{\substack{j \in J \\ j>i}}\left|b_{i j}\right|$

\section{d) Procédé de calcul}

(1) On part de

$$
\xi_{i} \quad, \quad I=\{i\} \quad, \quad \bar{I}=J /\{i\} \quad, \quad|I|=1
$$

l'on calcule $\varphi\left(\xi_{i}\right)=2 \cdot \sum_{\substack{k \in J \\ j \in J}}\left|b_{k j}^{\mathbb{\downarrow}}\right|$ 
L'on dispose ainsi d'un sommet initial et de la valeur de la fonction majorante en ce sommet.

(2) On parcourt la liste $Z$ des sommets. On choisit le sommet $s$ pour lequel la fonction majorante est maximum.

(3) Pour ce sommet on examine $I=I(s)$ :

i) Si $|I|=n$ ce sommet correspond à une solution unique qui est la solution optimale :

$$
\hat{q}=p \quad, \quad v(s)=f(\hat{q})
$$

ii) Si $|I|<n$ on passe à (4).

(4) On choisit un indice $i \in \bar{I}(s)$ selon un certain critère.

(5) Pour les deux valeurs possibles $q_{i}=1$ et $q_{i}=-1$ on calcule la fonction majorante.

On obtient deux nouveaux sommets $s_{1}$ et $s_{2}$, leurs valeurs $v\left(s_{1}\right)$ et $\varphi\left(s_{2}\right)$ et l'ensemble des choix effectués $p\left(s_{1}\right)$ et $p\left(s_{2}\right)$.

$I\left(s_{1}\right)=I\left(s_{2}\right)=I(s) \cup\{i\}$

$p\left(s_{1}\right)$ est tel que $p_{j}\left(s_{1}\right)=p_{j}(s) / j \in I(s), p_{i}\left(s_{1}\right)=1$

$p\left(s_{2}\right)$ est tel que $p_{j}\left(s_{2}\right)=p_{j}(s) ! j \in I(s), p_{i}\left(s_{2}\right)=-1$.

(6) Le sommet $s$ est exclu de la liste $Z$ des sommets et remplacé par les deux sommets $s_{1}$ et $s_{2}$.

L'on retourne à $(2)$.

\section{CONSIDERATIONS D'APPLICATION PRATIQUE}

\section{a) Calcul itératif de la fonction majorante}

Introduisons un vecteur $r(s)$ tel que :

$r_{k}(s)$ est indéterminé pour $k \in I$

$$
r_{k}(s)=\sum_{j \in I} b_{k j} p_{j} \quad \text { pour } \quad k \in \bar{I}
$$

On obtient que si $i$ est l'indice candidat

$$
\begin{gathered}
\varphi\left(s_{1}\right)=\varphi(s)+2\left[r_{i}(s)+\sum_{\substack{k \in \bar{I} \\
k \neq i}}\left|r_{k}(s)+b_{i k}\right|-\sum_{k \in \bar{I}}\left|r_{k}(s)\right|-\sum_{k \in \bar{I}}\left|b_{i k}\right|\right] \\
\varphi\left(s_{2}\right)=\varphi(s)+2\left[-r_{i}(s)+\sum_{\substack{k \in \bar{I} \\
k \neq i}}\left|r_{k}(s)-b_{i k}\right|-\sum_{k \in \bar{I}}\left|r_{k}(s)\right|-\sum_{k \in \bar{I}}\left|b_{i k}\right|\right]
\end{gathered}
$$


Définissons $K_{k}$, sous-ensemble de $\bar{I} /\{i\}$

$$
K=\left\{k \in \bar{I} /\{i\} / \operatorname{sgn}\left(r_{k}(s)\right)=\operatorname{sgn}\left(b_{i k}\right)\right\}
$$

et

$$
\bar{K}=\bar{I} / K /\{i\}
$$

Alors

$$
\begin{gathered}
\varphi\left(s_{1}\right)=\varphi(s)-4 \sum_{k \in \bar{K}} \min \left(\left|r_{k}(s)\right|,\left|b_{i k}\right|\right)+4 \min \left(\boldsymbol{r}_{i}(s), 0\right) \\
\varphi\left(s_{2}\right)=\varphi(s)-4 \sum_{k \in K} \min \left(\left|r_{k}(s)\right|,\left|b_{i k}\right|\right)+4 \min \left(-r_{i}(s), 0\right)
\end{gathered}
$$

et pour

$$
\begin{aligned}
& k \in \bar{I} /\{i\} \\
& r_{k}\left(s_{1}\right)=r_{k}(s)+b_{i k} \\
& r_{k}\left(s_{2}\right)=r_{k}(s)-b_{i k}
\end{aligned}
$$

Le calcul ne fait donc appel qu'à la valeur $\varphi(s)$, au vecteur $r(s)$ et à une partie de $i^{\mathrm{e}}$ ligne de la matrice $B$.

$\varphi(s)$ et $r(s)$ seront stockés pour chaque sommet $s$, mais $r(s)$ et $p(s)$ sont stockés simultanément sous forme d'un vecteur $t(s)$ :

$$
\begin{aligned}
t_{j}(s) & =p_{j}(s) / j \in I \\
t_{k}(s) & =r_{k}(s) / k \in \vec{I}
\end{aligned}
$$

\section{b) Choix de l'indice candidat}

Le choix a une grande influence sur le nombre de sommets qu'il faut considérer avant d'obtenir la solution. Différentes règles ont été testées. La meilleure s'est avérée être la suivante.

1) Réordonner d'abord la matrice $B$ en lignes et en colonnes pour obtenir que la suite des sommes des valeurs absolues des éléments de ces lignes et colonnes soit monotone décroissante.

2) Choisir lors de l'application de la méthode SEP l'indice suivant dans l'ordre naturel. Donc si $k$ indices ont déjà été considérés, l'indice candidat est le $k+1 \mathrm{e}$.

\section{c) Diminution du nombre de sommets en mémoire centrale}

1) Fonction minorante.

Par la méthode de l'optimum local classique de Thurstone il y a moyen de déterminer une solution qui sera fonction minorante : MIN. Tout sommet $s$ pour lequel $\varphi(s)<$ MIN est exclu de la liste $Z$. 
2) Troncature.

Le nombre de sommets en mémoire centrale est rapidement limité par la taille de l'ordinateur dont on dispose. Or la méthode SEP s'accommode très bien de troncatures.

On peut stocker en mémoire externe des sommets excédentaires et résoudre le problème sur l'arborescence tronquée, puis rappeler ces. sommets un à un pour terminer le travail.

\section{Ceci a deux avantages :}

- La liste des sommets dans laquelle il faut choisir le candidat est. plus courte; le choix est done plus rapide.

- Si dans l'arborescence tronquée on a trouvé une solution meilleure que l'optimum local du point (1) ci-dessus, le nombre de sommets des listes obtenues par les sommets rappelés sera plus petit.

\section{d) Résultats expérimentaux}

L'algorithme a été programmé en Fortran II pour une IBM 1620 - $40 \mathrm{~K}$ et pour des tailles de matrices ne dépassant pas $19 \times 19$. Dans les applications d'analyse factorielle la solution était généralement obtenue très rapidement : le nombre d'itérations. nécessaires était de l'ordre de $n$ à $3 n(n \times n$ taille de la matrice $)$. Une seule fois, dans le cas d'une analyse factorielle portant sur 19 variables, quand il s'agissait de déterminer le vecteur signe $\hat{q}$ d'un quatrième facteur éventuel qui n'était d'ailleurs plus statistiquement significatif le nombre d'itération était 256.

Coci est à comparer au nombre total d'itérations possibles qui est $2^{n}=2^{19} \simeq 500000$. En effet, si $B$ est une matrice nulle, l'optimum est. arbitraire, et si on désire obtenir toutes les solutions optimales, il faut. parcourir toute l'arborescence.

Les matrices de covariance résiduelles en analyse factorielle ont donc une structure telle que l'algorithme mène rapidement à la solution. L'optimum local obtenu par la méthode de Thurstone correspond alors à l'optimum global dans environ trois quarts des cas.

Sur l'ordinateur employé le temps d'une itération est de l'ordre de la seconde.

On a également essayé la méthode sur des matrices dont les éléments étaient choisis au hasard entre -1 et 1 . Dans ce cas le nombre d'itérations est beaucoup plus grand : de l'ordre de $2^{n / 2}$ (manque de structure).

Pour des applications en analyse factorielle avec des tailles de matrice de plus de $30 \times 30$, il est à craindre que le temps de calcul soit parfois prohibitif, même dans les ordinateurs récents. Il faudra alors se contenter d'un sous-optimum obtenu sur une arborescence tronquée. 


\section{EXEMPLE NUMERIQUE}

Un exemple numérique facilite la compréhension de la méthode.

Soit $B=\left[\begin{array}{rrrrrrr}0 & -2 & 1 & -3 & 5 & 4 & -1 \\ -2 & 0 & 4 & 2 & -5 & 2 & 3 \\ 1 & 4 & 0 & 1 & 2 & -3 & 4 \\ -3 & 2 & 1 & 0 & -1 & -2 & -3 \\ 5 & -5 & 2 & -1 & 0 & -5 & 6 \\ 4 & 2 & -3 & -2 & -5 & 0 & 1 \\ -1 & 3 & 4 & -3 & 6 & 1 & 0\end{array}\right]$

On cherche $\hat{q}$ qui maximise $q^{\prime} B q\left(q_{i}=+1\right.$ ou -1$)$

1)

$$
\bullet \max =\sum_{i} \sum_{j}\left|b_{i j}\right|=120=4 \cdot 30
$$

La nouvelle matrice ordonnée $C$ est :

$$
\left[\begin{array}{rrrrrrr}
0 & 6 & -5 & -5 & 5 & 2 & -1 \\
6 & 0 & 3 & 1 & -1 & 4 & -3 \\
-5 & 3 & 0 & 2 & -2 & 4 & 2 \\
-5 & 1 & 2 & 0 & 4 & -3 & -2 \\
5 & -1 & -2 & 4 & 0 & 1 & -3 \\
2 & 4 & 4 & -3 & 1 & 0 & 1 \\
-1 & -3 & 2 & -2 & -3 & 1 & 0
\end{array}\right]
$$

et $N 1=(5,7,2,6,1,3,4)$.

L'indice 5 original vient en première position, etc.

2) Pour cette matrice $C$ on recherche un optimum local.

Soit un vecteur d'essai $q^{\prime}=(1,1,1,1,1,1,1)$ par exemple les sommes $s_{i}$ sont $s^{\prime}=(2,10,4,-3,4,9,-6)$.

Le nouveau vecteur d'essai s'obtient en changeant le dernier signe :

$$
q^{\prime}=(1,1,1,1,1,1,-1)
$$

et $s^{\prime}$ devient $s^{\prime}=(4,16,0,1,10,7,6)$ 
$\checkmark$ min est donc

$$
\rho \min \sum_{i} s_{i}=44=4 \cdot 11
$$

3) Le sommet initial $s_{0}$ a la valeur $\frac{1}{4} \% \max =30$

$$
t^{\prime}\left(s_{0}\right)=(+, 6,-5,5,2,-1)
$$

On débute par le sommet initial.

4) On construit l'arborescence

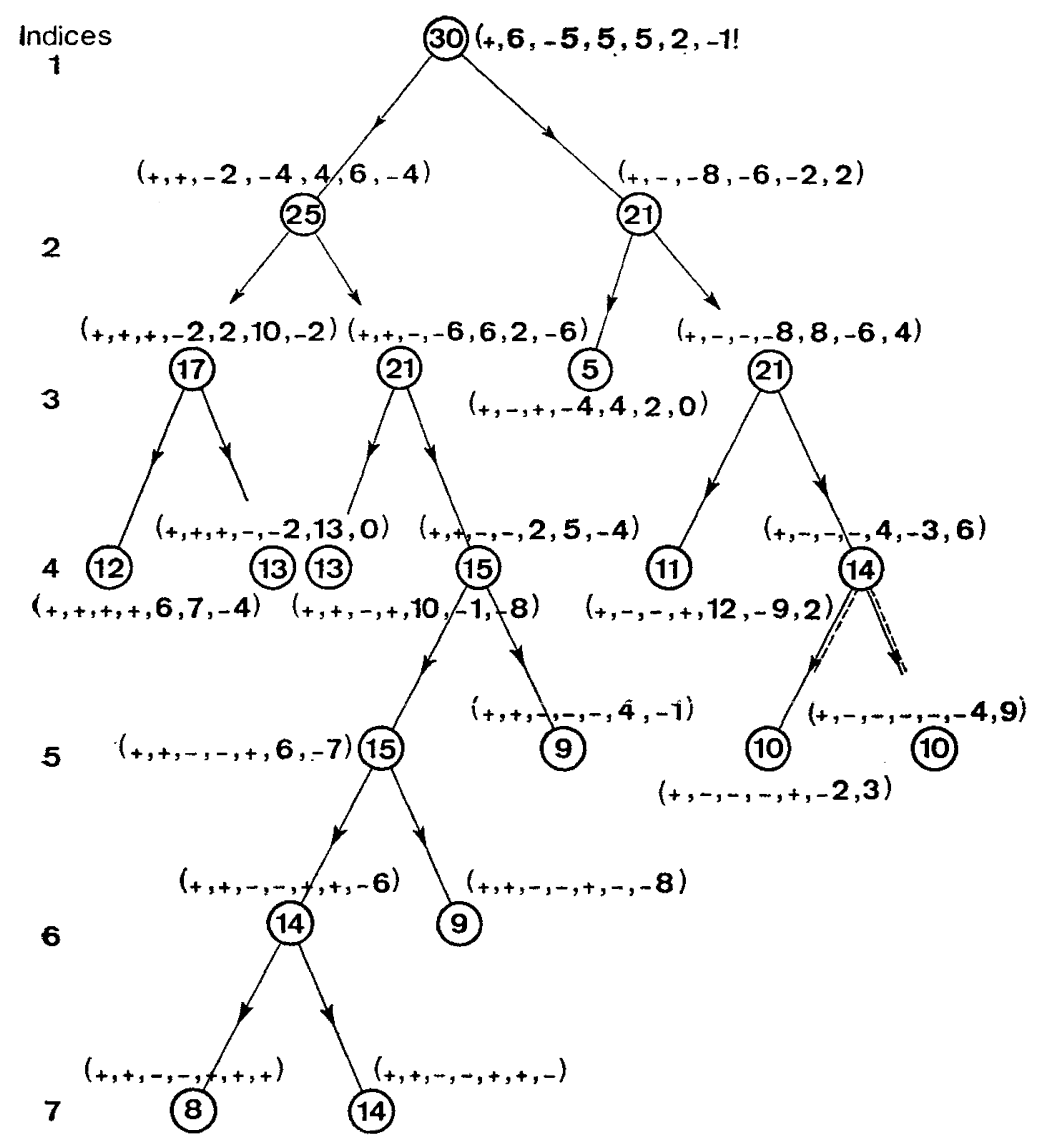

Pour obtenir deux nouveaux sommets à partir d'un sommet intermédiaire on effectue les opérations suivantes:

par exemple pour le sommet (25)

$$
t^{\prime}=(+,+,-2,-4,4,6,-4)
$$

et pour l'indice candidat $3: C_{3}=(||,, \mid, 2,-2,4,2)$ 
$\frac{1}{4} \Delta V\left(s_{1}\right)=+2+2+2+2=8$ (sommes des valeurs absolues des minima 2 à 2 des paires à signes opposés)

$\frac{1}{4} \Delta V\left(s_{2}\right)=4$ (sommes des valeurs absolues des minima 2 à 2 des paires à signes égaux).

On obtient ainsi deux sommets caractérisés par :

$$
\begin{array}{ll}
\frac{1}{4} \vartheta=25-8=(17) \quad, & t^{\prime}=(+,+,+,-2,2,10,-2) \\
\frac{1}{4} \vartheta=25-4=(21) & ,
\end{array}
$$

La méthode SEP donne la solution optimale en 9 à 10 itérations. L'itération en pointillé est inutile. En machine il y a une chance sur deux qu'elle soit exécutée (cela dépend de l'écriture formelle du programme).

Parmi les 11 sommets terminaux, 4 seulement seront gardés en mémoire : ceux pour lesquels $\frac{1}{4} \rho \geqslant 11=\frac{1}{4} \rho \min$

5) Le sommet terminal optimal a la valeur :

$$
\begin{aligned}
\frac{1}{4} \vartheta & =14 \\
\vartheta & =56=\hat{q} B \hat{q}
\end{aligned}
$$

le vecteur signe $t^{\prime}=(+,+,-,-,+,+,-)$.

En employant $N 1$, on retrouve l'ordre de la matrice originale $B$ $\hat{q}=(+,-,+,-,+,-,+)$

\section{DOMAINES D'APPLICATION DU PROBLEME DES SIGNES}

En dehors de la méthode centrö̈de d'analyse factorielle, le problème des signes peut avoir d'autres applications.

\section{a) Le problème des signes et les graphes}

Le problème des signes peut être représenté en tant que problème des graphes. En effet par changement de variables linéaires, tout comme au paragraphe 3 , le problème :

$$
\text { maximum } q^{\prime} B q \quad \text { sous } \quad q_{i}=+1 \text { ou }-1 \quad i=1, \ldots, n
$$

peut être ramené à :

$$
\text { maximum } y^{\prime} C y \text { sous } \begin{gathered}
y_{i}=0 \text { ou } 1 \quad i=1, \ldots, n \\
y_{0}=1
\end{gathered}
$$

où $C$ est une matrice $(n+1) \times(n+1)$. 
Considérons un graphe non orienté, complet, sans boucles à $n+1$ sommets numérotés de 0 à $n$. Chaque arête $(i, j)$ du graphe a pour valeur $C_{i j}=C_{j i}$.

Le problème des signes consiste à déterminer le sous-graphe complet comprenant le sommet 0 et pour lequel la somme des valeurs des arêtes est maximum. En effet $\hat{y}_{i}=1$ est équivalent à " le sommet $i$ appartient au sous-graphe ". L'arête $(i, j)$ n'appartient au sous-graphe que si $y_{i}=y_{j}=1$.

D'une façon analogue le problème des graphes suivant est équivalent au problème des signes.

Soit un graphe orienté ou non, complet ou non, avec ou sans boucles à $n$ sommets. Chaque arc $(i, j)$ a une valeur $\varphi_{i j}$.

On veut déterminer quel est le sous-graphe tel que la somme des valeurs des arcs appartenant au sous-graphe soit maximum.

Par le même procédé de changement de variables, ce problème est ramené au problème des signes de taille $n+1$.

\section{b) Exemple}

Un problème économique de transport.

Supposons qu'une compagnie de chemins de fer des États-Unis soit en faillite. Le gouvernement fédéral propose aux autres compagnies de reprendre tout ou partie du réseau de la compagnie défunte avec l'obligation pour une compagnie candidate d'assurer toute liaison entre deux gares reprises. Le réseau de la compagnie en faillite peut être représenté par un graphe sans boucles, dont les sommets sont les gares et les arêtes les voies reliant ces gares.

La compagnie est en faillite : il est donc certain que certaines liaisons sont déficitaires. La valeur d'une arête est le rendement (positif ou négatif) de la liaison. Le problème de la compagnie candidate est de déterminer le sous-graphe optimal, pour reprendre la partie du réseau la plus intéressante mais uniquement si le rendement du sous-graphe optimal est positif. Il suffit d'une arête à rendement positif pour qu'il existe un sousgraphe à rendement positif. Le rendement du graphe complet est négatif, puisque la première compagnie est en faillite. Le problème d'optimisation sera donc un vrai problème.

\section{REFERENCES}

[1] L. L. Thunstone, Multiple Factor Analysis, Univ. of Chicago Press, 1947.

[2] D. N. Lawley et A. E. Maxweld, Factor Analysis as a statistical method, Butterworths, London, 1963.

[3] P. Huard e.a., Mathématiques des programmes économiques, AFIRO, Dunod, 1964.

[4] B. Rox e.a., Programmes linéaires en nombres entiers et Procédure SEP, $M E T R A$, vol. IV, $\mathrm{n}^{\circ} 3,1965$.

[5] N. Agrs, Optimum seeking with Branch and Bound, Management Science, Dec. 1966 , vol. 13, nbr 4 . 